\title{
Optical trapping of air-borne light-absorbing particles with various laser beams
}

\author{
Porfirev A.P. \\ Samara State Aerospace University \\ Image Processing Systems Institute, Russian Academy of Sciences
}

\begin{abstract}
We demonstrate optical trapping carbon nanoparticle agglomerations in the air employing photophoretic forces. Three types of laser beams were used for optical trapping: a focused Hermite-Gaussian laser beam $\left(\mathrm{TEM}_{10}\right)$, an optical bottle beam and a hollow optical beam generated by Bessel beams superposition. The experimental results for each of the laser beams types are shown. Description of trapping features in each case is shown. Perspectives of application for each type of laser beams for three-dimensional optical manipulation are discussed.
\end{abstract}

Keywords: optical trapping, light-absorbing particles, Hermite-Gaussian beam, optical bottle beam, hollow optical beam

Citation: Porfirev A.P. Optical trapping of air-borne light-absorbing particles with various laser beams. Proceedings of Information Technology and Nanotechnology (ITNT-2015), CEUR Workshop Proceedings, 2015; 1490: 916. DOI: $10.18287 / 1613-0073-2015-1490-9-16$

\section{Introduction}

The optical manipulation of micro- and nanoscale objects with a laser beam was first demonstrated by A. Ashkin [1]. To date, this technique is widely used in the area of biophotonics and micromechanics for the manipulation of micro- and nano-objects [2-8]. Implementation of optical manipulation is possible in various environments such as in liquids or air. The causes of trapping are different depending on the medium and parameters of trapped objects. Therefore, the trapping of transparent micro-objects in liquids is typically carried out due to the action of the gradient force of a focused laser beam [9]. The trapping of light-absorbing micro-objects in air occurs through the action of photophoretic forces [10]. In the latter case, there are two phenomena present: positive photophoresis (particles move in a direction from the light source) and negative photophoresis (particles move towards the light source) [11].

This paper presents a comparison of the optical manipulation of light-absorbing carbon nanoparticle agglomerations using laser beams of three types: 1) a focused Hermite-Gaussian (HG) laser beam $\left(\mathrm{TEM}_{10}\right) ; 2$ ) an optical bottle beam (a beam with dark regions of exactly zero intensity surrounded by regions of higher intensity) [12]; 
and 3) a hollow optical beam that has zero intensity on the optical axis. Each of these beams has certain features that allows one to perform various types of manipulation.

\section{Optical manipulation with a Hermite-Gaussian laser beam (TEM10)}

In [13], it was shown that it is possible to carry out the three-dimensional trapping of non-spherical light-absorbing particles with a single focused Gaussian beam. This is possible through the action of the photophoretic force resulting from a different thermal accommodation coefficient that depends strongly on the surface state. In fact, the trapping of particles occurs in a region near the laser beam focus. Thus, it is possible to carry out independent multiple manipulation of light-absorbing particles by forming a plurality of intensity peaks in the focal plane.

The Hermite-Gaussian mode is described by the following well known expression [11]:

$$
\begin{aligned}
& E_{n m}(x, y, z)=i^{n+m}\left[\frac{w}{w(z)}\right]^{2} H_{n}\left[\frac{\sqrt{2} x}{w(z)}\right] H_{n}\left[\frac{\sqrt{2} y}{w(z)}\right] \times \\
& \exp \left[-\frac{x^{2}+y^{2}}{w^{2}(z)}+\frac{i k\left(x^{2}+y^{2}\right)}{2 R(z)}\right] \times \\
& \quad \exp \left[-i(n+m+1) \operatorname{arctg} \frac{z}{z_{0}}\right], \\
& \text { where } z_{0}=\frac{k w^{2}}{2}, w(z)=w\left[1+\left(\frac{z}{z_{0}}\right)^{2}\right]^{1 / 2}, R(z)=z\left[1+\left(\frac{z}{z_{0}}\right)^{2}\right],
\end{aligned}
$$

$w$ is the Gaussian beam waist, $R(z)$ is the Gaussian beam's wavefront curvature radius, $z_{0}$ is the Rayleigh range, $k$ is the wavenumber of light, $H n(x)$ is the Hermite polynomial.

Such beams are propagation-invariant (up to scale); that is, they keep structure of the transverse intensity distribution. Figure 1 shows the cross-section profiles of the intensity distribution for the different orders Hermite-Gaussian beams. We see that the order of a HG beam determines the number of generated intensity peaks, each of which can be used as an optical trap to capture light-absorbing particles in gaseous media.

a)
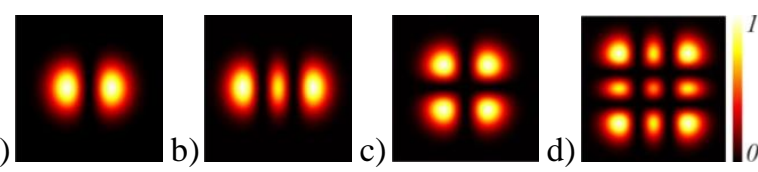

Fig. 1. - Cross-section profiles of the intensity distribution for Hermite-Gaussian laser beams: (a) TEM10, (b) TEM20, (c) TEM11, (d) TEM22

In optical trapping and optical guiding experiments, we use a laser $\operatorname{TEM}_{10}(\lambda=457$ $\mathrm{nm}$, with a maximum output power of $2000 \mathrm{~mW}$ ) (Fig. 8). An optical scheme of the experimental setup shown in Figure 2a. A generated beam is focused horizontally by a micro-objective $M O(3.7 \times, \mathrm{NA}=0.1)$ in the area inside the glass cuvette $C$ 
containing a suspension of micro-particles. Observation of the particle trapping is possible due to the scattered light recorded by the video camera Cam (Panasonic HDC-SD800, $1920 \times 1080$ pixels). A neutral density filter $F$ has been used to adjust the power of the laser beam.

a)

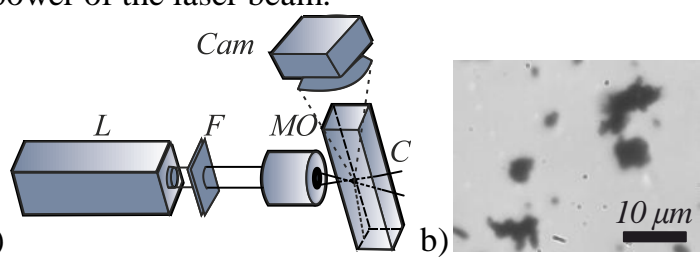

Fig. 2. - Optical manipulation experiment: (a) an experimental setup: $\mathrm{L}$ is a laser $\mathrm{TEM}_{10}$ $(\lambda=457 \mathrm{~nm}), \mathrm{F}$ is a neutral density filter, $\mathrm{MO}$ is a micro-objective $(3.7 \times, \mathrm{NA}=0.1), \mathrm{C}$ is a glass cuvette, Cam is a video camera Panasonic HDC-SD800 (1920 $\times 1080$ pixels); (b) carbon nanoparticle agglomerations used in experiment

To demonstrate the optical trapping and holding of absorbing particles, we used carbon nanoparticle agglomeration. The typical size of the agglomerations ranged to up to tens of micrometers (Figure 2b). The particles were sprayed with a syringe pump. Therefore, the particles initially had a significant acceleration directed to the bottom of the cuvette. The particles settled in the bottom of the cuvette under the influence of gravity. Some of them were trapped in the area of the laser beam. The guiding of the trapped particles was carried out by moving the micro-objective, which can be moved in three mutually perpendicular directions. As a result, movement of the focused laser beam results in movement of the trapped micro-particles.

Figure 3 shows the movement stage of two carbon nanoparticle agglomerations, each of which was trapped in the various intensity peaks of the HG TEM 10 laser beam. In this case, we carried out a controlled movement of the optically trapped micro-objects.

a)
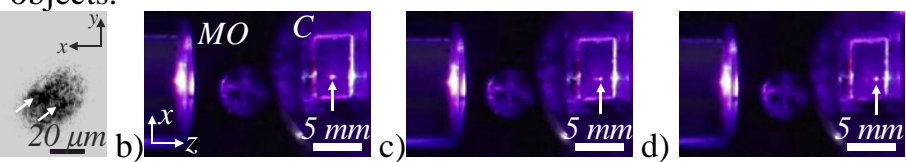

Fig. 3. - Optical trapping and guiding two carbon nanoparticle agglomerations with a HermiteGaussian TEM $_{10}$ laser beam: (a) trapped particles (denoted by white arrows); (b)-(d) movement stages of particles in plane $\mathrm{xz}$

Thus, the structure of the HG beams allows simultaneous, parallel threedimensional movement of several trapped light-absorbing micro-particles. The particles retain their relative position when moving in the space. These beams offer new opportunities for the controlled, multiple simultaneous manipulation of lightabsorbing non-spherical micro-objects. For example, it is possible to transport an array of light-absorbing particles, while their original location relative to each other will remain. Furthermore, this technique does not require the additional modulators to create multiple optical traps. 


\section{Optical manipulation with optical bottle beams}

Photophoresis phenomenon causes a light-absorbing object to move in a direction from the more heated to the less heated side of object. This makes it impossible to trap spherical micro-objects with a single Gaussian beam. The so-called optical bottle beams allow stable three-dimensional trapping of light-absorbing micro-particles to be carried out in gaseous media. An optical bottle beam is a beam with dark regions of exactly zero intensity surrounded by regions of higher intensity. For generation optical bottle beams, we used the method based on the formation of Bessel beams superposition [15]. This method enabled an optical bottle beam to be formed with a predetermined shape, such as a double optical bottle or optical bottle with a triangular profile of the intensity distribution.

For the experiments with optical bottle beams, we used the particles presented in Figure $2 \mathrm{~b}$. Figure 4 shows the optical scheme of the experimental setup. The laser beam is transformed by the diffractive optical element $D O E$ focused in the area inside glass cuvette by the micro-objective $M O(3.7 \times, \mathrm{NA}=0.1)$. Observation was carried out by a lens $L_{3}$. Adjustment of the output power of the laser beam allows us to vary intensity of formed traps and, therefore, change the values of the photophoretic forces acting on the trapped particles.

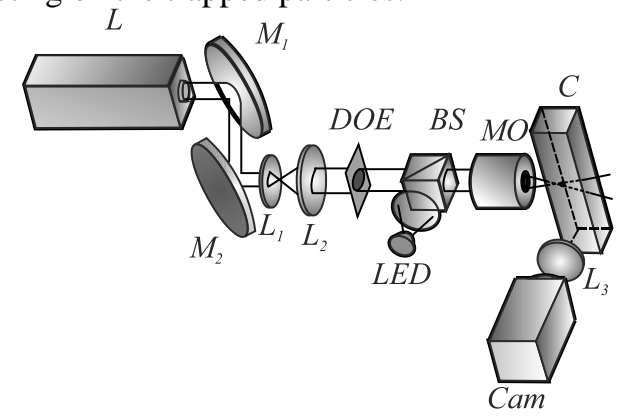

Fig. 4. - Experimental setup: $L$ is a solid-state laser $(\lambda=532 \mathrm{~nm}), M_{1}, M_{2}$ are mirrors, $L_{1}, L_{2}$, $\mathrm{L}_{3}$ are lenses, DOE is a diffractive optical elements that forms an optical bottle beam, BS is a beam splitter, LED is illuminating light-emitted diode, MO is a micro-objective $(3.7 \times, \mathrm{NA}=$ 0.1 ), $\mathrm{C}$ is glass cuvette, Cam is a CCD video camera

Figure 5 illustrates a typical trapping single carbon nanoparticle agglomeration with a single optical bottle. It can be seen that the trapped agglomeration remains in the optical trap during its transfer. We moved the optical trap by moving the focusing micro-objective $M O$, similar to experiments with a Hermite-Gaussian $\mathrm{TEM}_{10}$ laser beam. For this experiment, we have managed to move the agglomeration along the beam axis at distance of about $340 \mu \mathrm{m}$. The estimated value of the power within the boundaries of the optical bottle is about $16 \mathrm{~mW}$.

The methods described in [16], allow optical bottle arrays to form. The use of optical bottle arrays enables the boundary of trapping to be increased without changing the size of the traps themselves. As shown in [17], if the dimensions of the 
formed light traps and trapped micro-objects do not match, it is impossible to achieve stable trapping of micro-objects because they move uncontrollably inside the trap.

a)

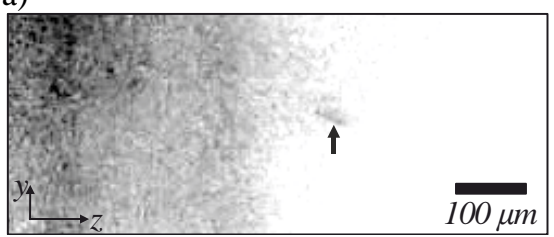

b)

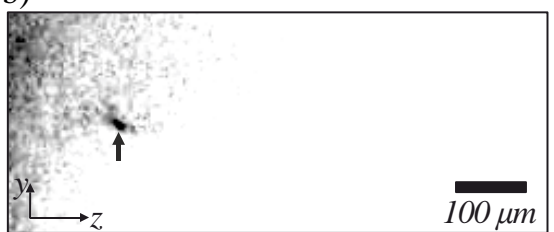

c)

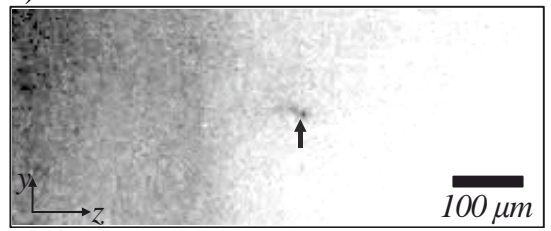

d)

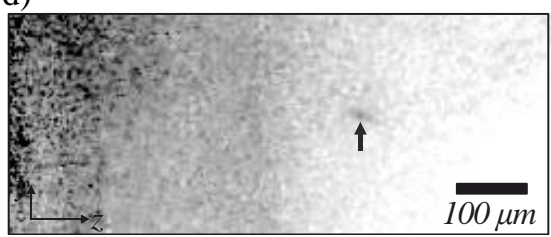

e)

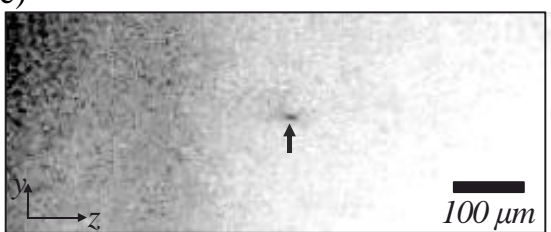

f)

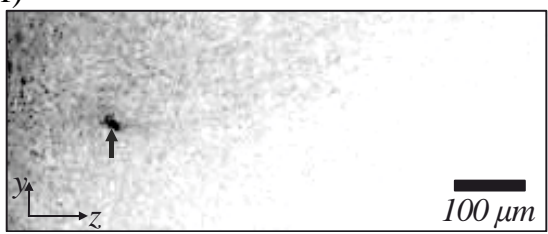

Fig. 5. - Experimental demonstration of trapping and guiding the carbon nanoparticles agglomeration with a single optical bottle. The black arrow points to the agglomeration

\section{Optical manipulation with hollow optical beams}

Hollow optical beams (HOBs) are optical beams with zero axial intensity along the propagation axis. These beams have diffraction-free properties on a limited interval along the optical axis. Due to such a configuration, the HOBs are universal optical traps to trapping both transparent and opaque micro-particles [18, 19].

There are various methods of forming such HOBs that have a predetermined crosssectional shape [20-24]. Changing the cross-sectional shape of the beams can change the shape of the region in which trapped particles will be moved.

In optical trapping experiments with HOBs, we used a solid-state laser $L(\lambda=532$ $\mathrm{nm}$, with a maximum output power of $500 \mathrm{~mW}$ ) (Figure 6). The laser beam was expanded with a telescope ( $L_{l}$ with $f_{l}=15 \mathrm{~mm}$ and $L_{2}$ with $f_{2}=35 \mathrm{~mm}$ ) to illuminate the DOE formed HOB. An airborne absorbing particle in a cuvette is trapped in the area of minimum intensity of the generated HOB. Observation of the particle trapping was possible due to the scattered light recorded by the video camera Cam (MDCE-5, $1280 \times 1024$ pixels $)$. The particles were imaged through a micro-objective $M O(8 \times$, $\mathrm{NA}=0.2$ ).

To demonstrate the optical trapping and holding of absorbing particles, we used carbon nanoparticle agglomeration as in the experiments described above (Figure $2 \mathrm{~b}$ ). Figure 7 shows the motion stages of a carbon nanoparticle agglomeration trapped by the HOB with an intensity distribution in the form of a regular pentagon contour. It 
can be clearly seen that the particle is also moved in a limited volume. At the time when the trapped particles remain inside the region of minimum intensity of the formed HOB, other particles settle on the bottom of the cuvette under the influence of gravity. Trapped particles do not leave the boundaries of the HOB because photophoretic force push them away from the region of maximum intensity in the region on the optical axis of the beam.

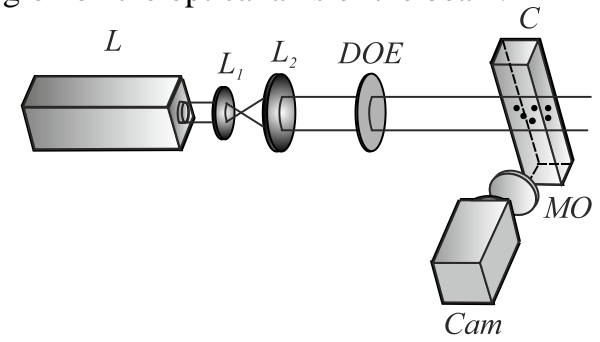

Fig. 6. - Experimental setup: $\mathrm{L}$ is a solid-state laser $(\lambda=532 \mathrm{~nm}), \mathrm{L}_{1}, \mathrm{~L}_{2}$ are lenses, DOE is a diffractive optical elements that forms a hollow optical beam, MO is a micro-objective ( $8 \times$, $\mathrm{NA}=0.2), \mathrm{C}$ is glass cuvette, Cam is a CCD video camera MDCE-5 (1280 $\times 1024$ pixels)

a)

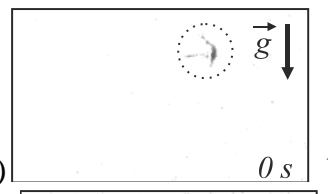

c)

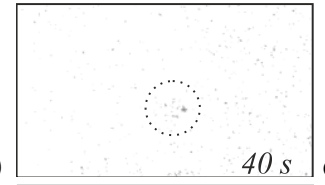

b)

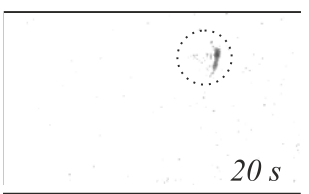

d)

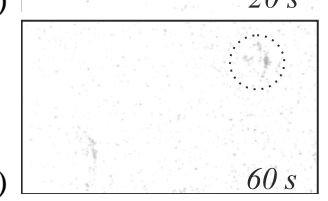

e)
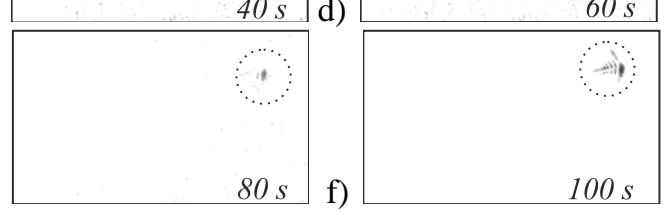

Fig. 7. - Optical trapping and holding of light-absorbing particles with HOB formed by DOE

The use of additional focusing optics enables the generation of converging HOBs, which have predetermined intensity distribution. It is therefore possible to form a complex spatial configuration of the optical beams. Each of these beams can be used to hold the particles in the region of desired size and shape. This 'storage' of microparticles can be used for contactless transport of microscopic objects. Furthermore, they can be used to study the interaction of various micro-objects trapped in a limited volume.

\section{Conclusion}

The paper describes the various ways to manipulate air-borne light-absorbing microscopic objects in air with laser beams of different types. The results of trapping

Information Technology and Nanotechnology (ITNT-2015) 
and manipulation experiments for each of laser beam types are shown. It is shown that:

1) With the use of Hermite-Gaussian beams, it is possible to achieve stable multiple trapping and guiding of light-absorbing non-spherical particles in generated separate intensity peaks;

2) With the use of optical bottle beams, it is possible to achieve stable trapping and guiding of the same particles at distances of hundreds of times their own dimensions;

3) With the use of hollow optical beams with a predetermined shape, trapping and holding light-absorbing particles can be carried out inside an area of predetermined shape within the area given shape, the dimensions of which exceed by ten times the particles' size.

None of these cases d require high-power lasers (enough power about 10-20 mW), due to the fact that photophoretic forces exceed the radiation pressure forces at the order of atmospheric pressure. [20].

\section{Acknowledgements}

This work was supported by the Ministry of Education and Science of the Russian Federation and Russian Foundation for Basic Research Grants No. 14-07-97038, No. 14-07-31291, No. 14-07-00177.

\section{References}

1. Ashkin A. Acceleration and trapping of particles by radiation pressure. Phys Rev Lett, 1970; 24(4): 156-159. doi: 10.1103/PhysRevLett.24.156

2. Grier DG. A revolution in optical manipulation. Nature, 2003; 424: 810-816. doi: 10.1038/nature01935

3. Dholakia K, Cizmar T. Shaping the future of manipulation. Nat Photonics, 2011; 5: 335342. doi: 10.1038/nphoton.2011.80

4. Kim K, Yoon J, Park YK. Simultaneous 3D visualization and position tracking of optically trapped particles using optical diffraction tomography. Optica, 2015; 2(4): 343346. doi: 10.1364/OPTICA.2.000343

5. Korobtsov A, Kotova S, Losevsky N, Mayorova A, Patlan V, Timchenko E, Lysov N, Zarubina E. Optical tweezers technique for the study of red blood cells deformation ability. Laser Phys, 2012; 22(7): 1265-1270. doi: 10.1134/S1054660X12070067

6. Morozov AA, Skidanov RV. Rotation of microturbine in compex vortex beams. Computer Optics, 2013; 37(2): 203-207. [in Russian]

7. Morozov AA, Skidanov RV. Complex vortex beams for of rotation of micromechanical elements. Computer Optics, 2013; 37(1): 68-75. [in Russian]

8. Skidanov RV, Rykov MA. The modificaction of laser beam for optimization of optical trap force characteristics. Computer Optics, 2013; 37(4): 431-435. [in Russian]

9. Ashkin A, Dziedzic JM, Bjorkholm JE, Chu S. Observation of a single-beam gradient force optical trap for dielectric particles. Opt Lett, 1986; 11(5): 288-290. doi: 10.1364/OL.11.000288

10. Shvedov VG, Desyatnikov AS, Rode AV, Krolikowski W, Kivshar YuS. Optical guiding of absorbing nanoclusters in air. Opt Express, 2009; 17(7): 5743-5757. doi: 10.1364/OE. 17.005743 
11. Keh HJ. Photophoresis of an aerosol sphere in a spherical cavity. Aerosol Air Qual Res, 2001; 1(1): 21-30.

12. Arlt J, Padgett MJ. Generation of a beam with a dark focus surrounded by regions of higher intensity: the optical bottle beam. Opt Lett, 2000; 25(4): 191-193. doi: 10.1364/OL.25.000191

13. Zhang Z, Cannan D, Liu J, Zhang P, Christodoulides DN, Chen Z. Observation of trapping and transporting airborne absorbing particles with a single optical beam. Opt Express, 2012; 20(15): 16212-16217. doi: 10.1364/OE.20.016212

14. Kogelnik H, Li T. Laser Beams and Resonators. Appl Optics, 1966; 5(10): 1550-1567. doi: 10.1364/AO.5.001550

15. Porfirev AP, Skidanov RV. Generation of optical bottle beams array by superposition Bessel beams. Computer Optics, 2012; 36(1): 80-90. [in Russian]

16. Porfirev AP, Skidanov RV. Generation of an array of optical bottle beams using a superposition of Bessel beams. Appl Optics, 2013; 52(25): 6230-6238. doi: 10.1364/AO.52.006230

17. Zhang P, Zhang Z, Prakash J, Huang S, Hernandez D, Salazar M, Christodoulides DN, Chen Z. Trapping and transporting aerosols with a single optical bottle beam generated by moiré techniques. Opt Lett, 2011; 36(8): 1491-1493. doi: 10.1364/OL.36.001491

18. Porfirev AP, Skidanov RV. Optical capture of microparticles in special traps. Computer Optics, 2012; 36(2): 211-218. [in Russian]

19. Kachalov DG, Pavelyev VS, Khonina SN, Skidanov RV, Porfirev AP. Experimental realisation of microparticle's optical trapping by use of binary radial DOE. Computer Optics, 2012; 36(1): 91-95. [in Russian]

20. Porfirev AP, Skidanov RV. A simple method of the formation nondiffracting hollow optical beams with intensity distribution in form of a regular polygon contour. Computer Optics, 2014; 38(2): 243-248. [in Russian]

21. Skidanov RV, Porfirev AP. Formation of optical beams with given intensity distribution in transverse plane for deposition and positioning of microscopic objects. Opt Mem Neural Net, 2014; 23(4): 233-239. doi: 10.3103/S1060992X14040080

22. Kovalev AA, Kotlyar VV, Porfirev AP, Kalinkina DS. Analysis of the orbital angular momentum of superposition of diffraction-free Bessel beams with a complex shift. Computer Optics, 2015; 39(2): 172-180. [in Russian]

23. Abramochkin EG, Kotova SP, Korobtsov AV, Losevsky NN, Mayorova AM, Rakhmatulin MA, Volostnikov VG. Microobject manipulation using laser beam with nonzero orbital angular momentum. Laser Phys, 2006; 16(5): 1. doi: 10.1134/S1054660X06050161

24. Porfirev AP, Skidanov RV. Formation of the massif of hollow beams for sedimentation and positioning of microparticles. Computer Optics, 2012; 36(3): 387-394. [in Russian] 